\title{
Estudio petrofísico de las areniscas del Palacio de Revillagigedo de Gijón (Asturias)
}

\author{
ROSA M. ESBERT Y LUIS VALDEON
}

Departamento de Petrologia. Facultad de Geología. Universidad de Oviedo

\section{$R E S U M E N$}

Se realiza el análisis y descripción de las caracteristicas petrográficas y fisicas de los materiales areniscosos, areniscas del Jurásico Superior, utilizadas en la construcción del Palacio de Revillagigedo de Gijón (1699-1711), incluyendo la cuantificación de algunos parámetros fisicos, propios de estos materiales, que tienen una especial significación en los procesos de alteración-alterabilidad, tales como: densidad de la roca, densidad de los granos minerales, porosidad total, porosidad abierta, succión capilar, etc.

Aplicando técnicas de porosimetria por inyección de mercurio se obtiene el valor de la porosidad accesible a dicho fluido, asi como los distintos intervalos de radios de acceso de poro responsables de esta porosidad que, junto con las observaciones realizadas por microscopia óptica y electrónica de los espacios vacios, ayudan a precisar la configuración del espacio poroso.

\section{$S U M M A R Y$}

The petrography and physical properties of the building stones used in the construction of the Revillagigedo Palace (Gijón, Spain), 1699-1711, are studied. The physical properties more closely related to the state of alteration and to the alterability processes of these Uper Jurassic sandstones are quantified, namely: rock density, porosity, open porosity, capillarity, etc.

The pore space configuration is appreciated under optic and electronic microscopy; the size distribution and total volume of the open pores are obtained by' means of mercury injection techniques.

\section{N T R O D U C CION}

Las areniscas utilizadas en la construcción del Palacio de Revillagigedo de Gijón exhiben una cierta multiplicidad de rasgos tipológicos, acordes con sus variaciones petrográficas que deben tomarse en consideración. Por ello y con el fin de que todas las variaciones presentes sean tenidas en cuenta, se ha realizado un amplio muestreo en el monumento - exterior e interior de la Colegiata, cuerpo central y torres del Palacio- extrayendo muestras que presentaban diferencias de: color, textura, tamaño de grano y tipo y grado de alteración; el conjunto muestreado queda representado por las variedades que en el trabajo se denominan A, B y C.

Desde el punto de vista geológico todas las muestras son areniscas pertenecientes al Jurásico detritico que aflora a lo largo de la costa este asturiana (Suárez Vega, 1974).

Se han muestreado también estos tipos en cantera eligiendo, dentro de la formación mencionada, dos lugares concretos de extracción, una cantera próxima a Somió (Gijón), variedad D y otra situada al oeste de Tazones, variedad E. 
Interesa resaltar que, el conocimiento de las caracteristicas petrográficas y físicas de los materiales rocosos utilizados en la construcción de monumentos, es imprescindible para afrontar cualquier trabajo relacionado con la deterioración actual o futura de cada monumento en cuestión ya sea para intentar esclarecer sus causas, formas y mecanismos de alteración o para acometer los tratamientos idóneos encaminados a limpiar, conservar o restaurar el monumento.

\section{PET R O G R A F I A}

Los estudios petrográficos están encaminados al análisis de las características mineralógicas y texturales de estos materiales areniscosos; se ha hecho especial énfasis en el estudio de la fracción mineral, la fase ligante o matriz y el medio poroso. Se han analizado, como ya se indicaba en la Introducción, muestras procedentes del Palacio de Revillagigedo, extraidas de diversas partes del edificio y muestras procedentes de cantera.

\section{Caracterización mineralógica}

Mediante la ayuda de diversas técnicas microscópicas, ópticas y electrónicas, se ha llegado a la conclusión de que son rocas con una gran uniformidad mineralógica; están compuestas esencialmente por cuarzo y filosilicatos.

El cuarzo (71-82 \%) es el principal elemento detrítico y a su vez el mineral más abundante. Aparece en granos de muy diversas formas: irregulares con bordes a veces angulosos y otras redondeados, también idiomórficos, sobre todo los de menor tamaño.

Los filosilicatos forman el armazón o matriz de la roca (3-18 \%) aunque también pueden encontrarse en cristales aislados con tendencia idiomórfica $(0,5-1,4 \%)$; son de origen secundario $\mathrm{y}$ en ocasiones, por un reemplazamiento incompleto, permite la identificación del mineral original que suele ser ortosa o moscovita.

Los feldespatos potásicos (1,3-8,4\%) están presentes en proporciones reducidas; suelen ser ortosa y/o microclina, aunque es más abundante la ortosa.

Las plagioclasas son muy escasas; su porcentaje, junto al de algunas micas sin identificar y accesorios tipo circón, turmalina y opacos, varía del 0,1 al $1,2 \%$.

Los fragmentos líticos son también escasos y de naturaleza diversa aunque los más frecuentes son los agregados policristalinos, tipo chert.

Los óxidos e hidróxidos de hierro suelen teñir al resto de constituyentes minerales dando al conjunto, en visión macroscópica, una coloración rojiza más o menos intensa.

Para una mejor caracterización e identificación de los filosilicatos se han realizado diagramas de polvo orientados de la fracción menor de $0,05 \mathrm{~mm}$, mediante difractometria de Rayos X, obteniéndose además los porcentajes mineralógicos de cada constituyente. (Tabla I).

Los porcentajes más altos de minerales arcillosos corresponden a las rocas de cantera. La especie más abundante es la caolinita que aparece en forma de láminas superpuestas, tal como se aprecia en la figura 1. La illita es menos abundante; aparece sólo en dos de las muestras del monumento y está ausente en las muestras de cantera. En la figura 2 puede observarse un conjunto de cristales de illita, con su peculiar aspecto fibroso. 
T A B LA I

Tipos y porcentajes de filosilicatos de la fracción arcillosa

\begin{tabular}{|l|c|c|c|c|c|}
\hline \multicolumn{1}{|c|}{ Filosilicatos \% } & A & B & C & D & E \\
\hline Caolinita & $0-1$ & 4,5 & 7,5 & 17 & 16 \\
Illita & $3-5$ & 4,5 & - & - & - \\
Mica (sin determinar) & - & - & 6,75 & - & - \\
Metahalloisita & - & - & 0,75 & - & - \\
Clorita-Vermiculita & - & 1 & - & 20 & 20 \\
\hline \% TOTALES & 3,6 & 10 & 15 & & - \\
\hline
\end{tabular}

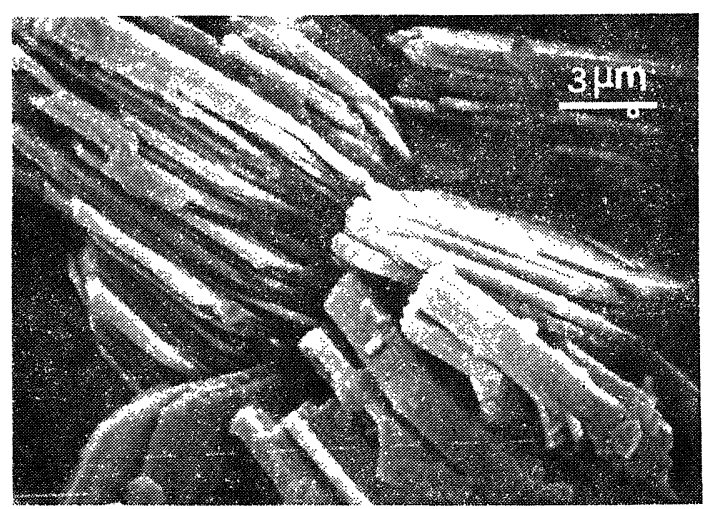

Fig. 1.--Caolinita euhedral observada al microscopio electrónico de barrido S.E.M.

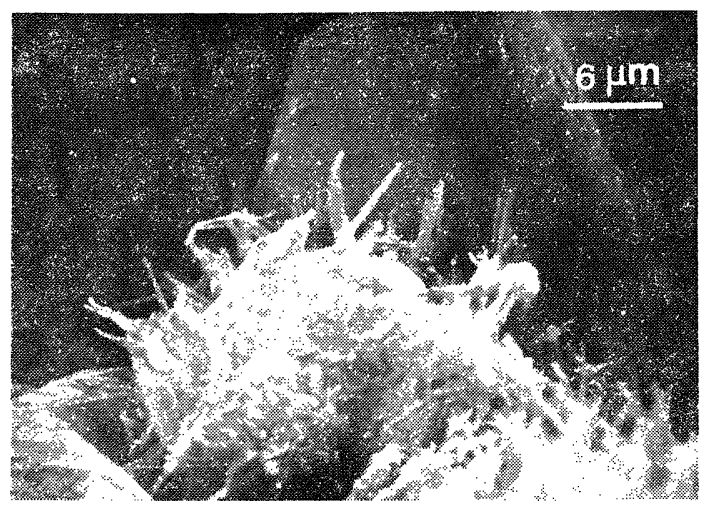

Fig. 2.-Aspecto de los cristales de illita. al microseopio electrónico de barrido S.E.M.

\section{Caracterización textural}

Desde el punto de vista textural las rocas muestran dos partes claramente diferenciables: los granos minerales y la matriz.

Los granos minerales presentan, en el conjunto de muestras, una cierta heterometría tal como reflejan los histogramas de la fig. 3. Se encuentran variedades cuyo tamaño oscila de muy fino a grueso $(0,062 \mathrm{~mm}$ a $0,625 \mathrm{~mm})$ (muestras A y D) y otras en las que el tamaño oscila de muy fino a medio $(0,062 \mathrm{~mm}$ a $0,5 \mathrm{~mm})$ (muestras B, C y E), (Blatt, 1982). Figuras 4 y 5.

En cuanto a la cantidad de matriz arcillosa hay diferencias entre las muestras estudiadas; algunas de ellas tienen porcentajes de matriz de alrededor del $10 \%$; suelen ser las muestras del monumento. En otras muestras la proporción de matriz crece alcanzando valores próximos al $17 \%$, son las variedades de cantera. Dentro de esta matriz arcillosa, y como puede verse en la Tabla I, el mineral mayoritario es la caolinita, siguiéndole, en proporción mucho menor la illita y otras micas sin determinar.

Respecto a su disposición, la matriz, suele presentarse de dos modos diferentes, agrupada en zonas, figura 6, o en forma pelicular alrededor de los granos minerales, figuras 7 y 8 . En las variedades en las que se agrupa en zonas, crece el número de contactos entre los granos, los cuales son mayoritariamente homofásicos, cuarzo-cuarzo, figura 9. 
Debido a los menores porcentajes de feldespatos y micas, los contactos heterfásicos cuarzofeldespato, cuarzo-mica y mica-feldespato son muy escasos.
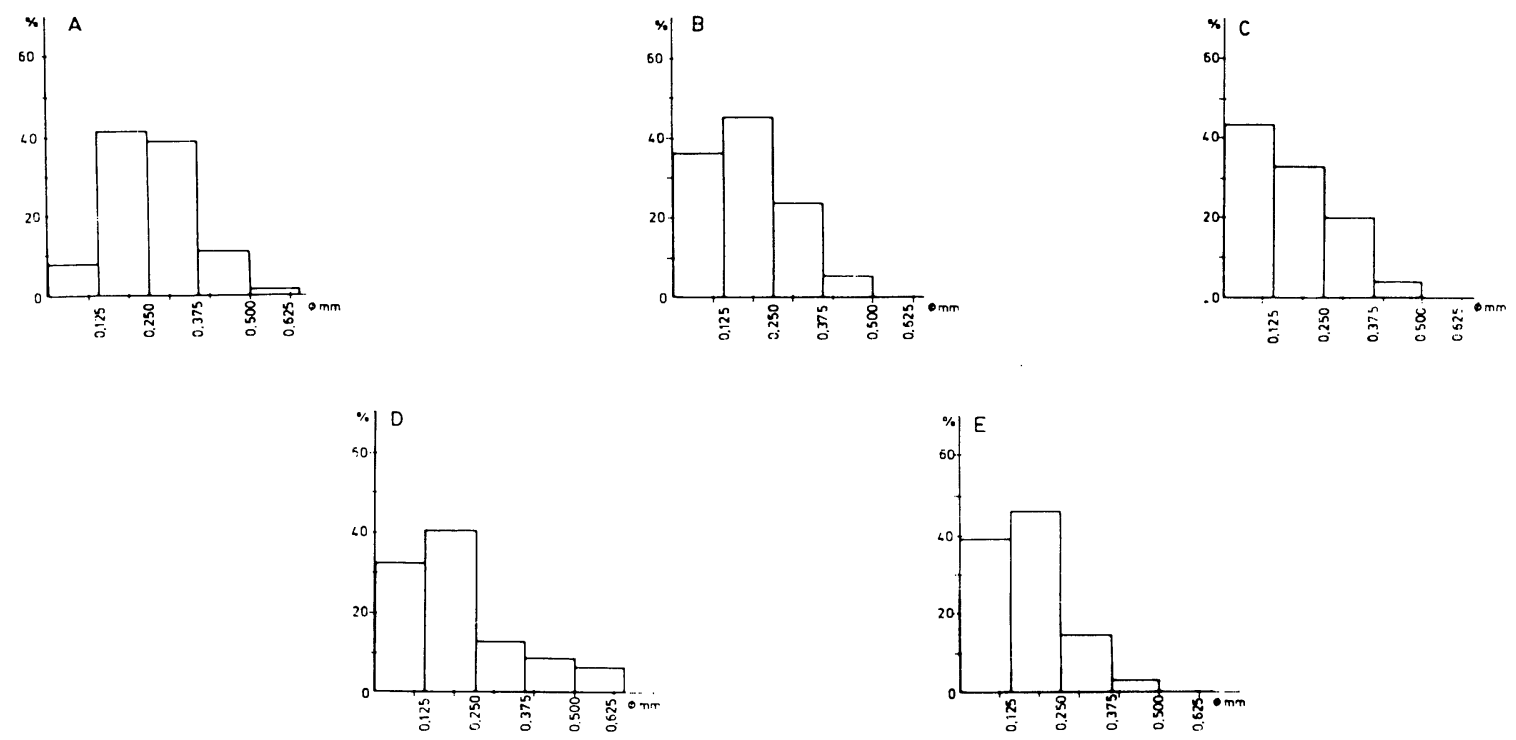

Fig. 3.-Histogramas de variación del tamaño de grano, en las diferentes muestras estudiadas.
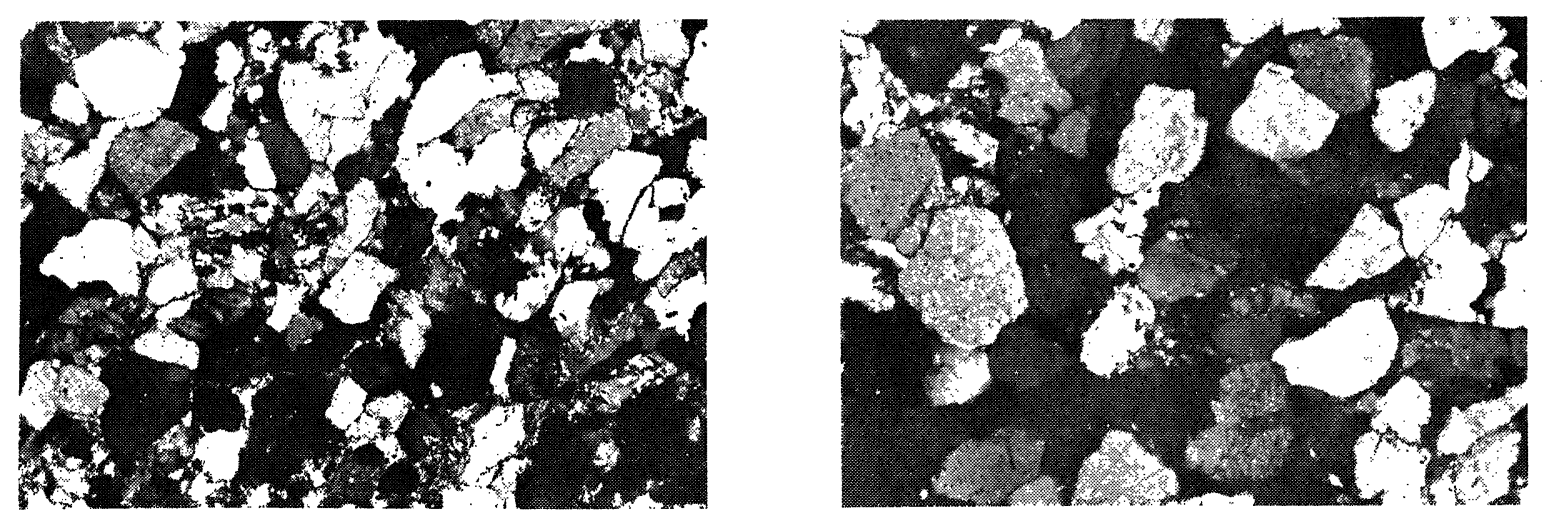

Figs. 4 y 5.-Aspecto textural del conjunto rocoso. al microscopio de polarización. Se aprecian las diferencias en el tamaño de grano de una a otra micrografia. N.C. $\times 34$.
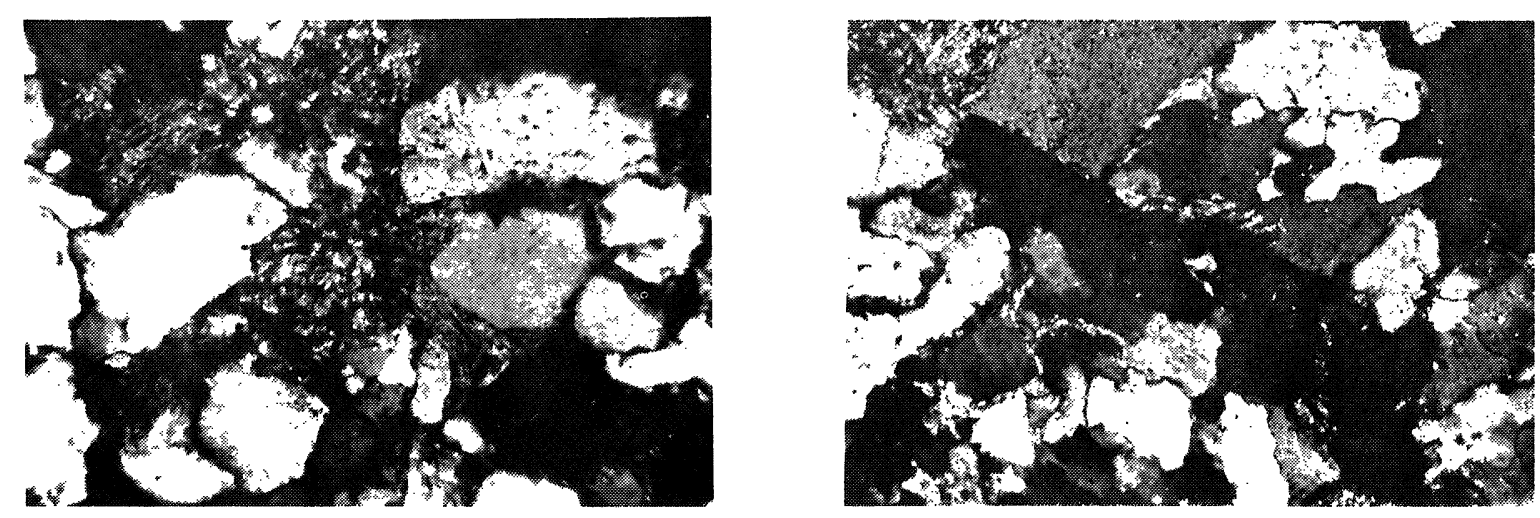

Figs. 6 y 7.-Matriz filosilicatada, agrupada en zonas, foto 6 , N.C. $\times 73$ o distribuida en forma pelicular. alrededor de los granos minerales, foto 7 , N.C. $\times 155$. 


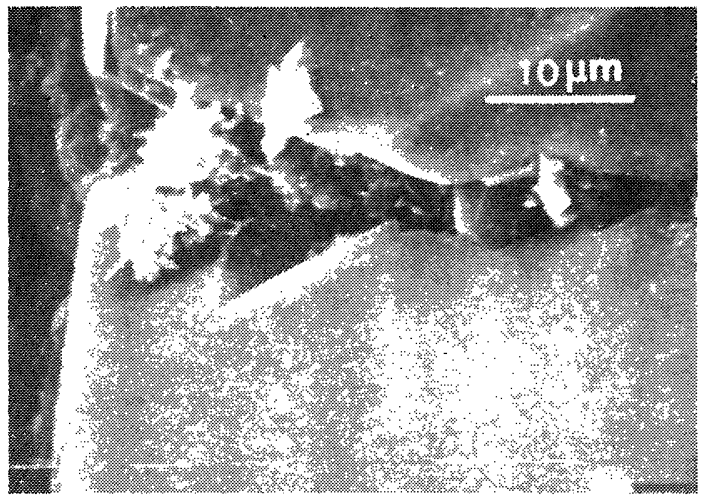

Fig. 8.-- Detalle de la matriz pelicular. al microscopio electrónico S.E.M.

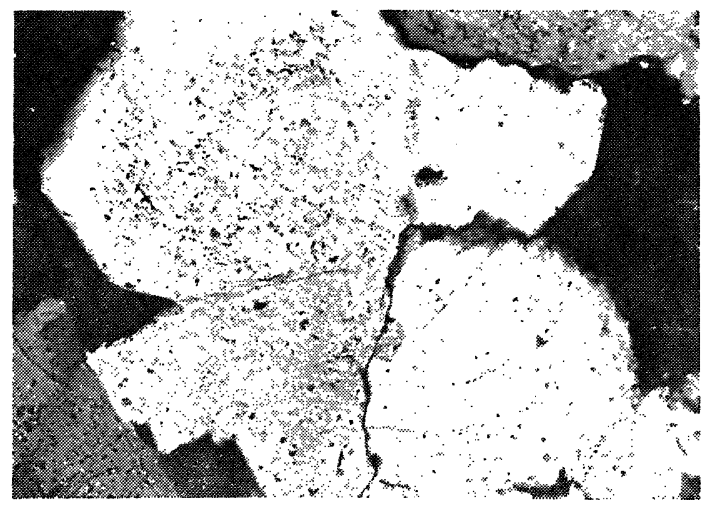

Fig. 9.-Aspecto de los contactos tangenciales. cuarzocuarzo $\mathrm{NC} \times 95$

\section{Descripción, cuantificación y configuración del medio poroso}

Otro de los constituyentes texturales que ha sido analizado es el medio poroso, que en estas rocas areniscosas, adquiere singular importancia. (Esbert et al, 1984).

Los poros presentan morfologias diversas, la mayoria son redondeados, aunque los hay también elongados; a veces tienen bordes lisos, otras veces angulosos, figuras 10 y 11 . Suelen aparecer en el seno de los granos minerales como cuarzo y ortosa, asi como a lo largo de los contactos entre granos, en ocasiones interconectados con fisuras inter o intragranulares, figuras 12 y 13; también debe considerarse la especial configuración de la matriz; los minerales filosilicatados, que la constituyen, caolinita e illita presentan hábitos cristalográficos que favorecen la existencia de múltiples espacios vacios, figura 14.

Los tamaños de los espacios vacios son variables; hay microporos (radio de acceso $<7,5 \mu \mathrm{m}$ ) y macroporos (radio de acceso $>7,5 \mu \mathrm{m}$ ). Ambos configuran, junto con las microfisuras, el espacio macro y microporoso de estos materiales, el cual se cuantifica más adelante. Las microfisuras son abundantes; por lo general, son abiertas, con aberturas de anchura variable, siempre de unas pocas micras, las hay inter, intra y transgranulares; las intergranulares son las más frecuentes y suelen localizarse en los contactos cuarzo-cuarzo, ya que éste es el mineral que más abunda en estas rocas, fig. 15.

Para una mejor caracterización del medio poroso, se han realizado ensayos de porosimetria por inyección de mercurio, con lo que se ha logrado tener una idea aproximada de cómo este espacio poroso se desarrolla tridimensionalmente. Se ha cuantificado la porosidad accesible al mercurio, porosidad comunicada, obteniéndose las curvas de distribución de dicha porosidad, en función del radio de acceso de los poros. Para ello se ha efectuado una doble inyección de mercurio para cada muestra ensayada con el fin de diferenciar la porosidad "atrapada", es decir, la que corresponde a poros con difícil acceso, de la porosidad efectiva. Es un hecho constatable que cuando inyectamos mercurio en una muestra rocosa, entre la primera y segunda inyección, hay siempre una histéresis más o menos notoria la cual es debida a poros o fisuras que presentan un dificil acceso, entradas tortuosas y en los que el mercurio, penetrado a presión, difícilmente puede salir. (Bousquie, 1979).

El porosimetro propiamente dicho, permite determinar el volumen de mercurio que, para cada intervalo de presión pre-fijado, penetra en los poros o espacios vacios con radios de acceso comprendidos entre $0,0037 \mu \mathrm{m}$ y $7,5 \mu \mathrm{m}$, a los que se ha denominado microporos. El "aparato de llenado" permite averiguar el volumen de mercurio que penetra en los poros con un radio de acceso entre $7,5 \mu \mathrm{m}$ y $70 \mu \mathrm{m}$, macroporos. 


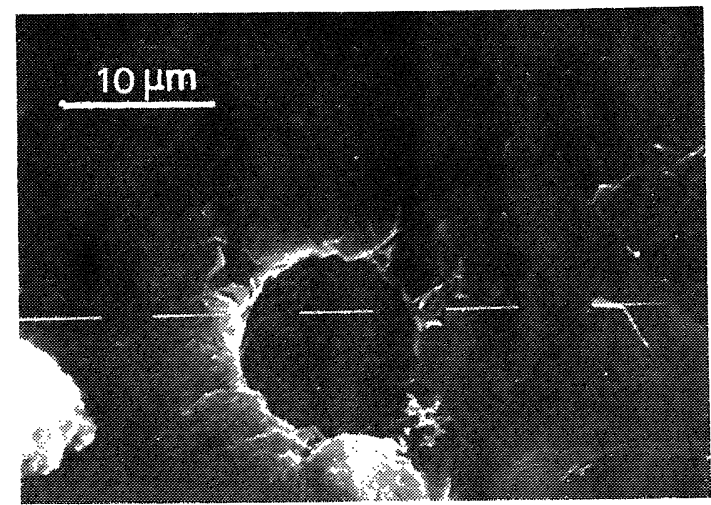

10

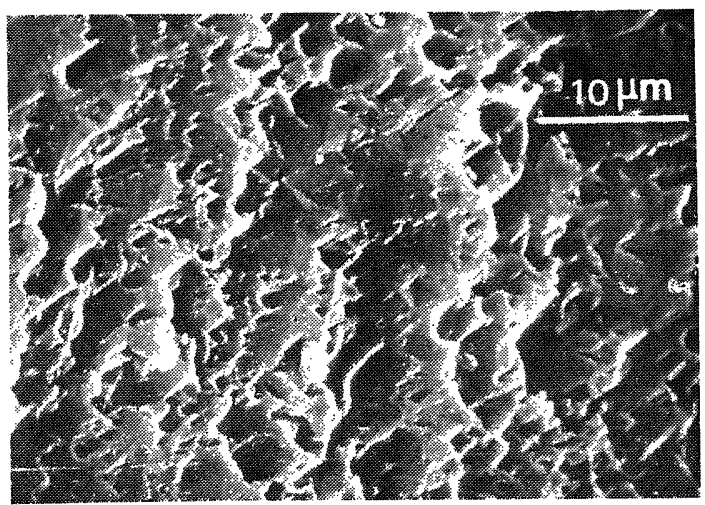

12

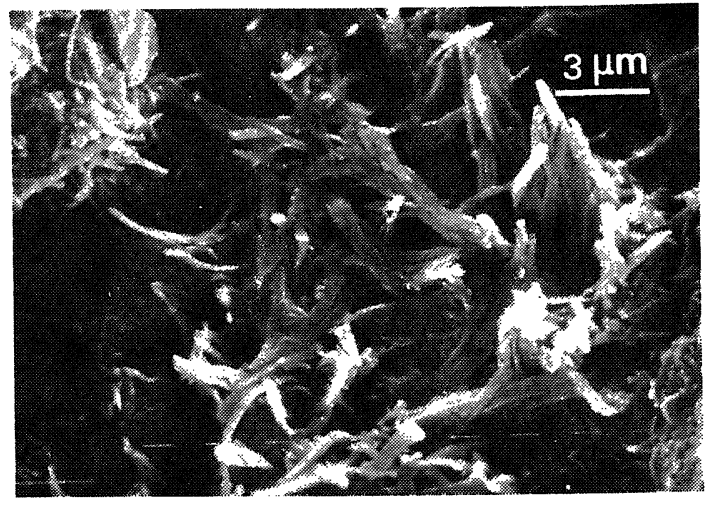

14

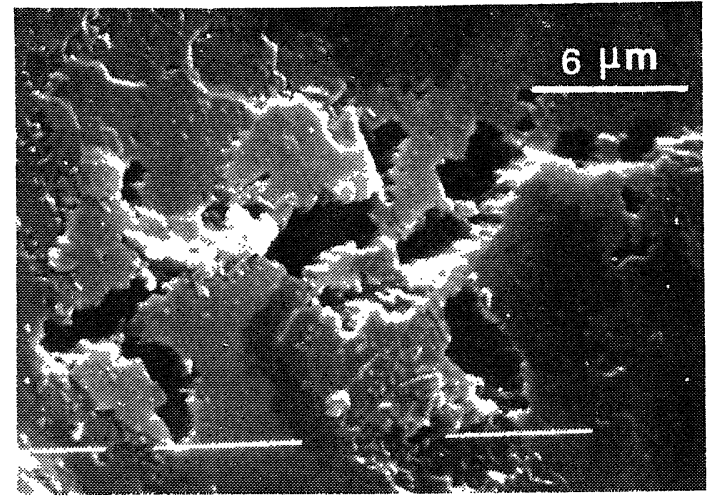

11

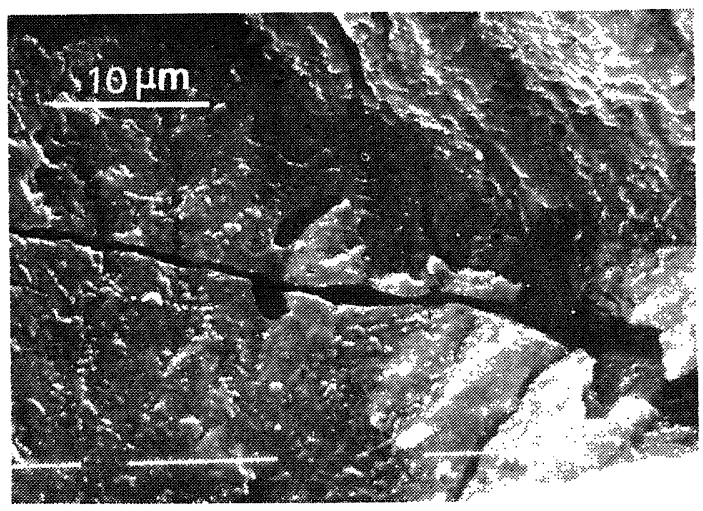

13

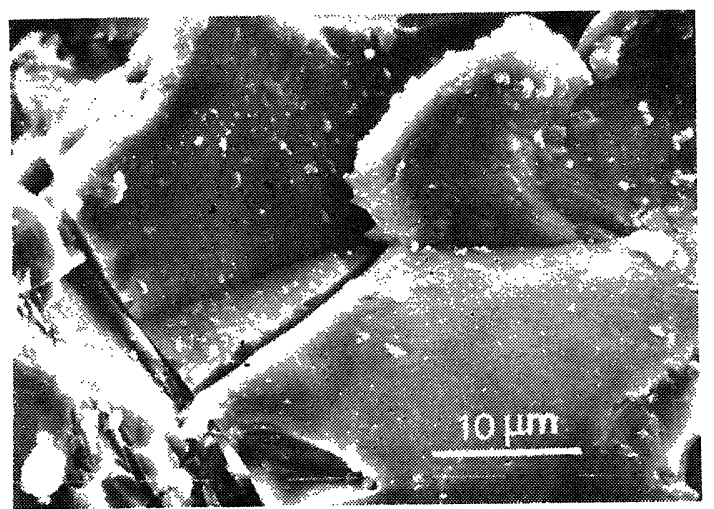

15

Figs. 10 a 15.-Diversos aspectos del medio poroso, al microscopio electrónico de barrido, S.E.M. 10. Poro redondeado en el seno de un cristal de cuarzo. 11. Poros diversos, con tendencia elongada. 12. Multitud de poros de formas y tamaños variados en un cristal de ortosa. 13. Poros interconectados con microfisuras. 14. Aspecto de los espacios vacios existentes entre los filosilicatos de la matriz. 15. Fisuras transgranulares abiertas entre granos de cuarzo.

La evolución de la microporosidad, evidenciada por el volumen de mercurio introducido en los microporos, se ha obtenido gráficamente mediante una curva continua gracias a un registrador complementario adosado al porosimetro.

Con los resultados se ha elaborado la Tabla II donde aparecen, para cada muestra ensayada, los valores de porosidad comunicada $\left(n_{0}\right)$, los de macro y microporosidad, asi como los valores correspondientes de porosidad atrapada, macro y microporosidad atrapada. 
TABLA II

Valores de porosidad obtenidos por porosimetría de inyección de mercurio

\begin{tabular}{|c|c|c|c|c|c|c|}
\hline Muestra & $\begin{array}{c}\mathbf{n}_{\mathbf{0}} \\
\mathbf{( \% )}\end{array}$ & $\begin{array}{c}\text { Macroporosidad } \\
(\mathbf{r}>\mathbf{7 , 5} \boldsymbol{\mathbf { m }}) \\
\mathbf{( \% )}\end{array}$ & $\begin{array}{c}\text { Microporosidad } \\
(\mathbf{r}<\mathbf{7 , 5} \boldsymbol{\mu} \mathbf{~ m}) \\
\mathbf{( \% )}\end{array}$ & $\begin{array}{c}\text { Porosidad } \\
\text { atrapada } \\
\mathbf{( \% )}\end{array}$ & $\begin{array}{c}\text { Macroporosidad } \\
\text { atrapada } \\
\mathbf{( \% )}\end{array}$ & $\begin{array}{c}\text { Microporosidad } \\
\text { atrapada } \\
\mathbf{( \% )}\end{array}$ \\
\hline A & 18,3 & 11,0 & 7,3 & 9,4 & 4,8 & 4,7 \\
B & 17,4 & 12,8 & 4,5 & 10,3 & 8,4 & 2,0 \\
C & 15,4 & 8,05 & 7,4 & 9,4 & 7,2 & 2,2 \\
D & 13,5 & 0,5 & 13,05 & 6,4 & 0,3 & 6,1 \\
E & 10,6 & 1,1 & 9,5 & 7,5 & 1,8 & 5,5 \\
\hline
\end{tabular}

El examen comparativo de los diferentes valores de la Tabla II permite resaltar algunos aspectos referidos a los distintos tipos de porosidad y configuración del espacio poroso en el conjunto de areniscas estudiadas. Así, la porosidad accesible al mercurio, porosidad comunicada, que oscila entre un $10,6 \%$ y un $18,3 \%$, presenta valores más altos en las muestras del monumento (A, B y C) que en las de cantera (D y E).

En cuanto a los valores de macro y microporosidad se marcan claramente dos tendencias. En una de ellas hay porcentajes significativos tanto de macro como de microporosidad, pero los primeros resultan superiores, son las piedras del monumento. La otra tendencia la muestran las piedras de cantera, en las que el porcentaje de microporosidad es muy elevado, representa más del $90 \%$ de la porosidad comunicada; son rocas casi exclusivamente microporosas.

Los valores de porosidad atrapada son en todas las muestras superiores al $50 \%$ de la porosidad comunicada, con lo que se pone de manifiesto que, en el medio poroso, un elevado porcentaje de poros tienen difícil acceso y, por tanto, una alta capacidad de retención de fluido, siempre que éstos sean capaces de penetrar en los mismos. A su vez, dentro de la porosidad atrapada es posible diferenciar entre macro y microporosidad atrapada (Tabla II). Del examen de sus valores se deducen dos comportamientos: uno representado por las muestras $\mathrm{D}$ y $\mathrm{E}$, en que prácticamente toda la porosidad atrapada es microporosidad, y el otro reflejado por las muestras A, B y $\mathrm{C}$ en las que la porosidad atrapada es atribuible preferentemente a macroporos.

Se han elaborado las respectivas curvas porosimétricas acumuladas - fig. 16- referidas a: presión de inyección, volumen de poros penetrados por el mercurio y radio de acceso de los mismos, las cuales ayudan a visualizar las caracteristicas porosimétricas y tendencias de cada muestra ensayada (Esbert y Marcos, 1983). En ellas pueden observarse los dos comportamientos antes reseñados, piedras del monumento $-\mathrm{a}, \mathrm{b}$ y $\mathrm{c}-\mathrm{y}$ piedras de cantera $-\mathrm{d}$ y e-

En el grupo marcado por la primera tendencia resalta el gran volumen de macroporosidad respecto al total de porosidad comunicada y la brusca inflexión de las curvas de la primera inyección al pasar del tramo macro al microporoso (fig. 16, a, b y c). En el segundo grupo no se observa dicha inflexión al pasar de uno a otro tramo, el volumen microporoso es mucho mayor (fig. 16, d y e).

Con los datos de estas curvas se han confeccionado los histogramas correspondientes, que se muestran en la figura 17, a, b, c, d y e. En ellos puede apreciarse la implicación que cada intervalo de radio de acceso de poro tiene en la porosidad comunicada de las muestras. 

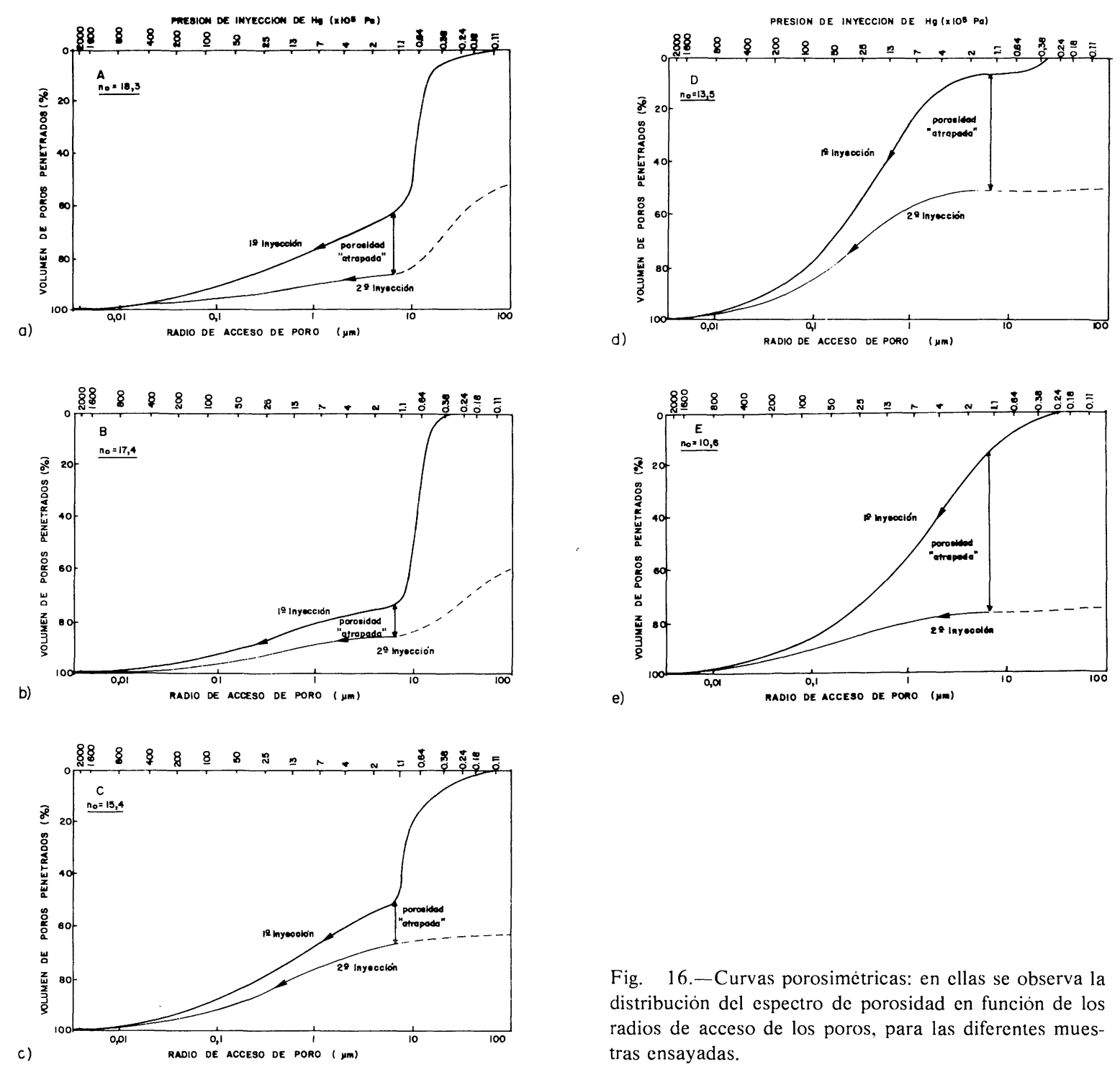
Fig. 16.-Curvas porosimetricas: en ellas se observa la
distribución del espectro de porosidad en función de los
radios de acceso de los poros, para las diferentes muestras ensayadas.

\section{Propiedades físicas}

Con el fin de caracterizar, desde el punto de vista físico, los materiales pétreos del Palacio de Revillagigedo, se han determinado una serie de propiedades fisicas elementales, como: Densidad de los granos minerales $\left(\rho_{\mathrm{s}}\right)$; Densidad aparente de la roca seca $\left(\rho_{\mathrm{d}}\right)$; Porosidad abierta $\left(n_{0}\right)$; Porosidad total $(n)$; Grado de relleno de los poros $\left(S_{\mathrm{r}}\right)$ y Coeficiente de capilaridad $(C)$.

Dichas propiedades se han calculado según las normas estandarizadas por la I.S.R.M. (1979), excepto para el coeficiente de capilaridad, calculado, únicamente para muestras de cantera y según normas francesas (N.F. B.-10.502). En la Tabla III, se recogen los valores hallados.

De la observación de la tabla se deduce que la densidad de los granos minerales, $\rho_{s}$, es prácticamente idéntica para todas las variedades rocosas siendo el valor medio obtenido de $2,65 \mathrm{~g} / \mathrm{cm}^{3}$. Esto es lógico si se recuerda que estas rocas son mayoritariamente cuarzosas.

Los valores de densidad de la roca seca, $\rho_{\mathrm{d}}$, muestran poca variabilidad; oscilan entre 2,03 y $2,25 \mathrm{~g} / \mathrm{cm}^{3}$, y son inversamente proporcionales a los valores de porosidad tanto abierta como total, lo que resulta evidente ya que, las rocas con más porosidad, tendrán, lógicamente, menos densidad. 


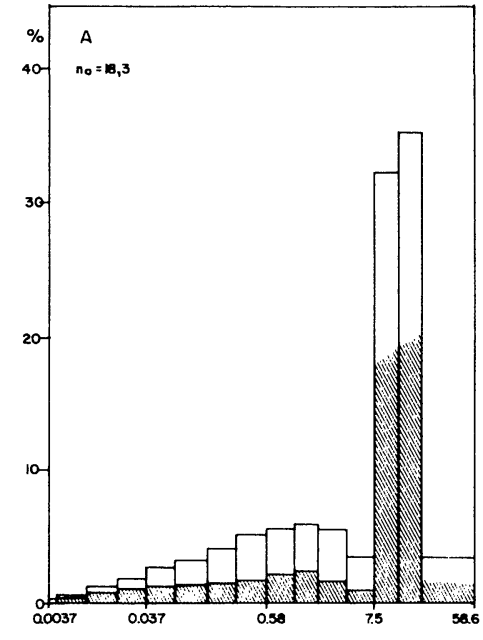

a)

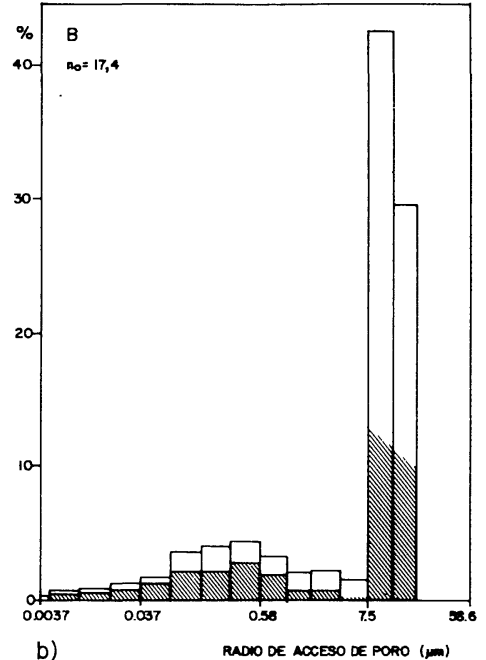

b)

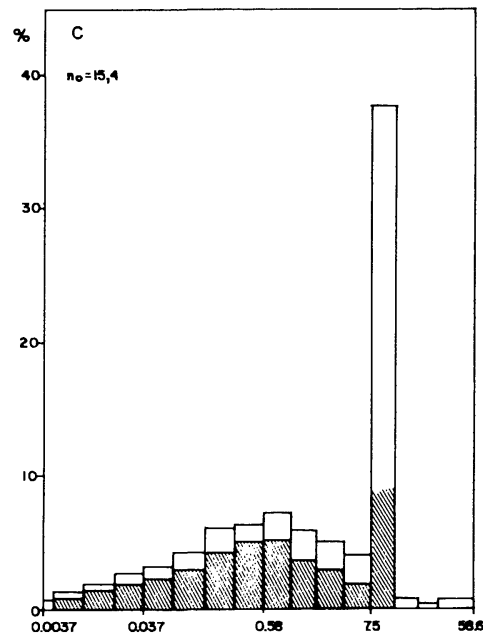

c)

RADNO DE ACEESO DE POAO (m)

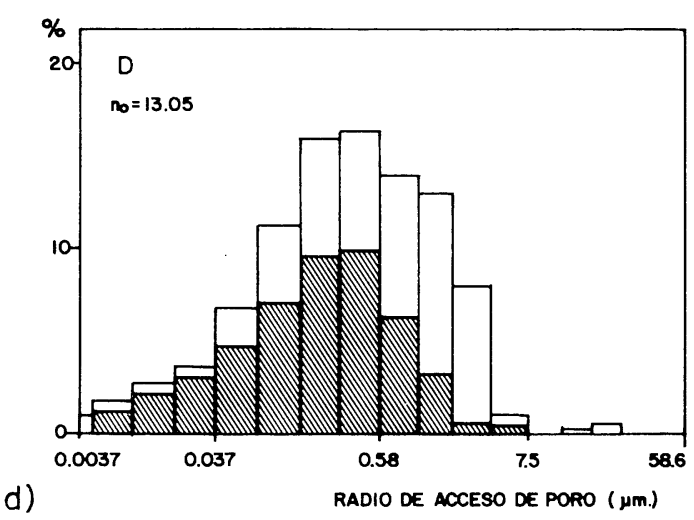

Fig. 17.-Variación del volumen de poros penetrados por el mercurio en relación con el radio de acceso de los mismos, en las diferentes muestras ensayadas. Las zonas ralladas corresponden a la porosidad atrapada.

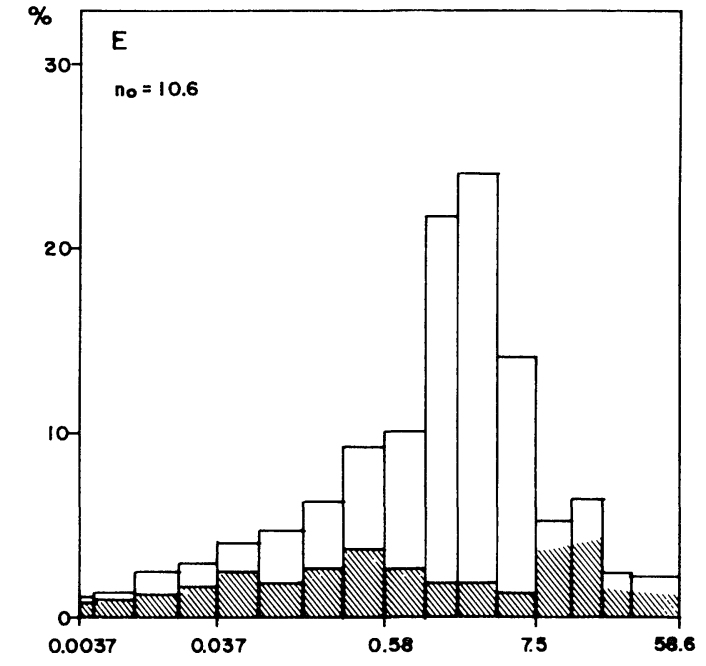

e)

T ABLA III

Valores obtenidos para las diferentes propiedades fisicas determinadas

\begin{tabular}{|c|c|c|c|c|c|c|}
\hline Muestra & $\begin{array}{c}\rho_{\mathrm{s}} \\
\left(\mathrm{gr} / \mathrm{cm}^{3}\right)\end{array}$ & $\begin{array}{c}\rho_{d} \\
\left(\mathrm{gr} / \mathrm{cm}^{3}\right)\end{array}$ & $\begin{array}{c}n_{0} \\
(\%)\end{array}$ & $\begin{array}{c}n \\
(\%)\end{array}$ & $\begin{array}{l}S_{r} \\
(\%)\end{array}$ & $\begin{array}{c}\mathrm{C} \\
\mathrm{kg} / \mathrm{m}^{2} \min ^{0.5}\end{array}$ \\
\hline A & 2,65 & 2,03 & 22,50 & 23.30 & 96.50 & - \\
\hline B & 2,66 & 2.07 & 18,50 & 22,20 & 83,30 & - \\
\hline $\mathrm{C}$ & 2,66 & 2.18 & 16,30 & 18,00 & 90,80 & -- \\
\hline $\mathrm{D}$ & 2,65 & 2.25 & 14.30 & 15,10 & 94,90 & 71 \\
\hline $\mathrm{E}$ & 2.66 & 2,14 & 18.40 & 18.90 & 97.50 & 32 \\
\hline
\end{tabular}

La porosidad total $(n)$ tiene valores elevados para este tipo de rocas areniscosas y de forma especial para las rocas del monumento, en las que llega al 23,3\%, siendo la media de estas muestras (A, B y C), del 21,2\%. Sin embargo, en las muestras de cantera (D y E) la media de la porosidad total resulta algo menor, alrededor del $17 \%$. 
La porosidad abierta $\left(n_{0}\right)$ es, en todos los casos, inferior a la porosidad total $(n)$, pero los valores se hallan muy próximos, por lo que se deduce que la mayoría de los poros están comunicados. Esto se destaca mejor con la medida de $S_{\mathrm{r}}$ o grado de relleno de los poros, que relaciona el volumen de poros comunicados $\left(n_{0}\right)$, con el volumen total de poros $(n)$; se aprecian porcentajes muy altos en todas las muestras con una media superior al $92 \%$, de volumen de poros ocupados.

Las medidas de porosimetría de mercurio nos evidencian el mismo hecho: los valores de porosidad comunicada - porosidad abierta - obtenidos mediante esta técnica son muy similares a los que refleja la Tabla III, excepto en la muestra D (Tazones) cuyos valores, sensiblemente más bajos, hacen pensar que hay poros cuyos tamaños de radio de acceso no han podido ser analizados por el porosimetro con el que se realizaron los ensayos (S-200, Carlos Erba).

En la determinación del coeficiente de capilaridad (C) (Mamillan, 1981), referido a las muestras de cantera, se han obtenido valores muy dispares poniéndose de manifiesto, en todos los casos, la alta capilaridad de las rocas de Somió respecto a las de Tazones. En la figura 18 se muestra la relación entre la masa de agua absorbida por unidad de superficie y el tiempo, en ambas variedades rocosas. Las pendientes ayudan a separar fácilmente los diferentes comportamientos.

En las figuras 19 y 20 puede visualizarse, sobre las probetas de ensayo de las variedades Somió

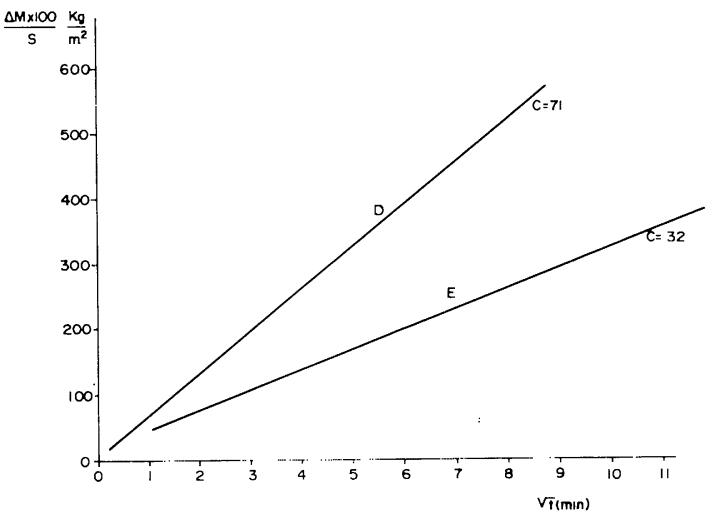

Fig. 18. - Masa de agua absorbida por capilaridad. por superficie especifica. con relación a $\sqrt{t} \cdot C=$ Cocficiente de capilaridad.
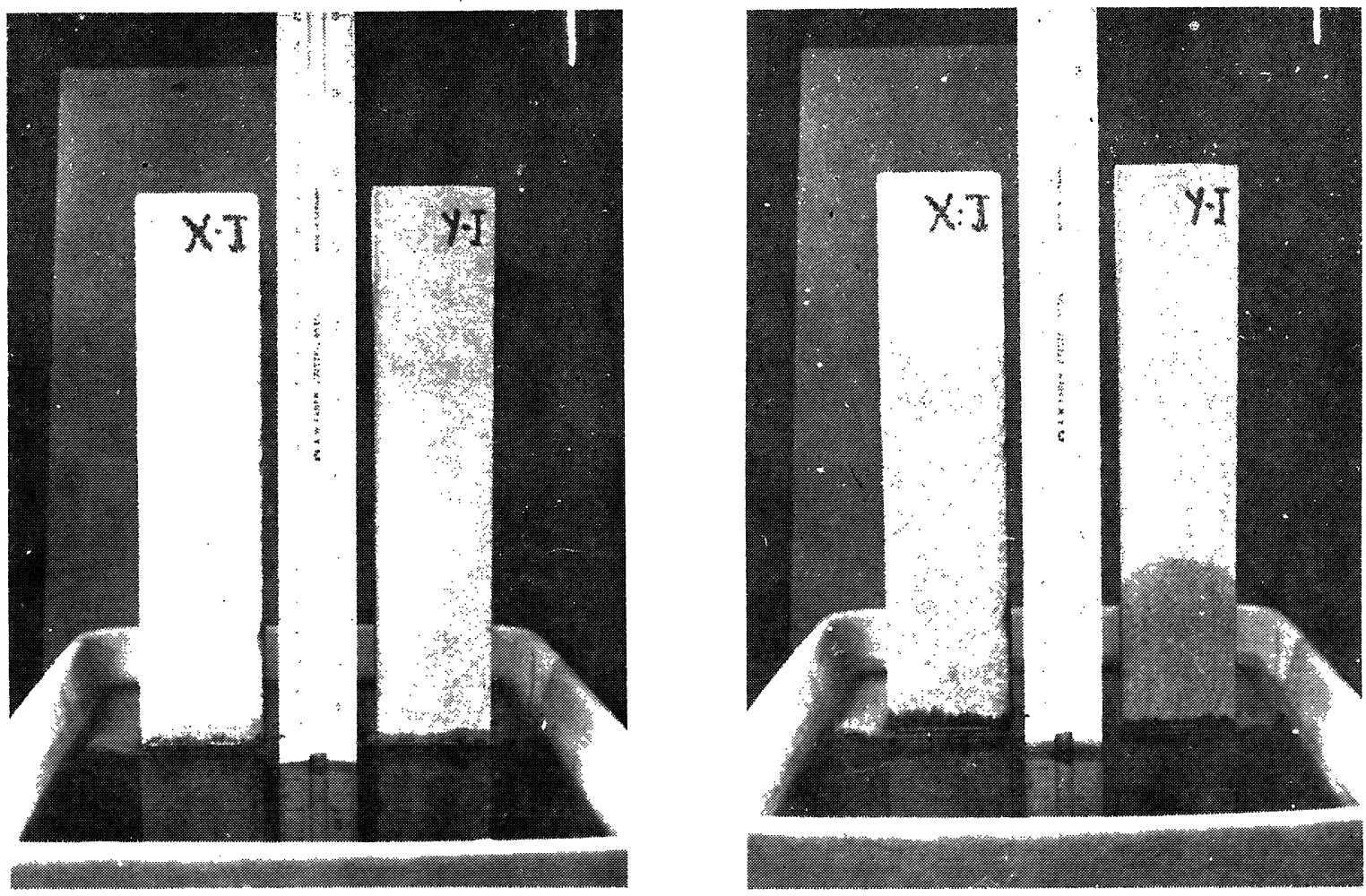

Figs. 19 y 20.- Alturas alcanzadas por el agua en las variedades de Somio (probetas X) y Tazones (probetas Y) a los cinco minutos y nueve horas respectivamente. de iniciado el ensayo de succión capilar. 
(izquierda, X) y Tazones (derecha, Y), la diferencia de alturas alcanzadas por el agua en dos momentos diferentes y alejados entre sí del ensayo. Concretamente, la figura 19 se realizó a los cinco minutos del comienzo de ensayo y ya es apreciable la mayor altura alcanzada por el agua en la probeta de Somió (X). Esta diferencia se acentúa a medida que transcurre el ensayo; así en la figura 20 tomada a las nueve horas del comienzo, puede verse con claridad que la altura del agua en la probeta de Somió es aproximadamente el doble de la alcanzada en la de Tazones.

\section{CONCLUSIONES}

- Los materiales de construcción utilizados en el Palacio de Revillagigedo de Gijón han sido siempre areniscas extraídas, con toda probabilidad, de los tramos areniscosos del Jurásico Superior, Mälm, que afloran al Este de Gijón.

- Desde el punto de vista petrográfico estas areniscas están compuestas esencialmente por cuarzo y filosilicatos, cuyos porcentajes, considerados conjuntamente, representan de un $78 \%$ a un $92 \%$ de la composición global de las rocas. Los filosilicatos, que integran fundamentalmente la matriz arcillosa, son caolinita e illita. La relación porcentual granos minerales/matriz arcillosa varia en las distintas muestras; por lo general, las muestras de cantera tienen porcentajes más elevados de matriz (Tabla I).

- Los espacios vacíos (poros y microfisuras) son abundantes; se localizan en el seno de la matriz filosilicatada, en el interior de los granos minerales y a lo largo de los contactos cuarzo-cuarzo.

- En cuanto a los valores de porosidad se ha podido precisar que: la porosidad total en el conjunto de materiales muestra valores altos $-15 \%$ a $23 \%-$, dentro de los rangos de porosidad establecidos para este tipo de rocas (5\% - $20 \%$, Farmer, 1968); un porcentaje elevado de estos poros están comunicados como nos lo demuestran los valores obtenidos por porosimetria de mercurio que oscilan entre un 10,6 y un $18,3 \%$. Estos ensayos nos muestran también que en el conjunto se marcan dos tendencias claramente definidas: en una de ellas, rocas del monumento, más del $75 \%$ de la porosidad comunicada es atribuible a macroporos $(r>7,5 \mu \mathrm{m})$. En la otra tendencia, muestras de cantera, aproximadamente el $95 \%$ de la porosidad comunicada es debida a microporos $(r<7,5 \mu \mathrm{m})$. Todas las variedades rocosas presentan porcentajes elevados de porosidad atrapada.

- En las muestras en que predomina la macroporosidad, ésta se distribuye en un intervalo reducido de radio de entrada de poros, entre 7,5 y 19,9 $\mu \mathrm{m}$. (Fig. 17 a, b, c). En las muestras con elevada microporosidad, ésta es atribuible a un rango mucho más amplio de entrada de poro, de 0,0037 a $7,5 \mu \mathrm{m}$ (Fig. 17, d, e) cubriendo, por tanto, todo el espectro microporosimétrico.

- El tipo de porosidad, asi como sus respectivos porcentajes es importante conocerlo, por cuanto condiciona los procesos de alteración y la alterabilidad futura de los materiales rocosos (Goñi et al., 1970); (Walker et al., 1969).

- En el conjunto de rocas estudiadas puede establecerse una clara correlación entre propiedades fisicas y caracteristicas petrográficas (Montoto, 1983): La densidad de la roca $\left(\rho_{d}\right)$, en cada variedad, está en proporción inversa a su porosidad; si se examinan los diferentes valores hallados puede verse que en ellos influye también el distinto porcentaje de filosilicatos de cada muestra. Cuando éste crece en detrimento de las otras especies minerales, se incrementa ligeramente el valor de la densidad de la roca, ya que los filosilicatos, sobre todo los que forman la matriz, caolinita e illinita, tienen unos valores de densidad algo mayores que los del cuarzo y los feldespatos. 
- Tanto el porcentaje como el tipo de porosidad, macro o microporosidad, están decisivamente influenciados por tres caracteristicas petrográficas: relación porcentual, granos minerales, matriz filosilicatada, tipo de espacios vacíos - macro o microporos- y tamaño de grano y grado de heterometria granular. Asi, en las diferentes variedades areniscosas, el porcentaje de filosilicatos, que mayoritariamente forman la matriz, varía notablemente; en concreto varía de las muestras del monumento - \% de filosilicatos entre $3,6 \%$ y $15 \%$ - a las de cantera -\% de filosilicatos en torno al $20 \%$ - También un grupo y otro de piedras difieren en cuanto al tipo de porosidad; las muestras del monumento tienen valores altos de macroporosidad, las de cantera son esencialmente microporosas.

- Esto puede deberse, junto al tamaño medio de grano y heterometría granular, a que en las rocas con porcentajes más elevados de matriz, los filosilicatos suelen tapizar los espacios vacios de diferente naturaleza que configuran la red porosa, reduciendo sus dimensiones. Ello también explica que dichas rocas no sólo muestren un dominio de la microporosidad sobre la macroporosidad, sino que además, sean las que menos porosidad abierta tienen.

- En cuanto a la capilaridad, calculada para las muestras de cantera, la variedad de Somió tiene un valor mucho más elevado que la de Tazones $\left(C=71 \mathrm{~kg} / \mathrm{m}^{2} \min 0.5\right.$ y $32 \mathrm{~kg} / \mathrm{m}^{2} \min 0,5$, respectivamente), lo cual puede relacionarse no sólo con los valores absolutos de porosidad, sino también con las características del medio poroso; las muestras de Somió tienen porcentajes de microporosidad más altos que las de Tazones y es sabido que, cuanto más pequeños sean los poros, mayor será la fuerza de ascensión capilar y por lo tanto la altura alcanzada por el agua (Mamillan, 1981).

\section{A G R A D E C I M I EN T OS}

A la Comisión Asesora de Investigación Cientifica y Técnica (Ministerio de Educación y Ciencia) que financió la presente investigación (Proyecto n. ${ }^{\circ} 4446-79$ ).

\section{B I B L I O G R A F I A}

BLATT, H., (1982): "Sedimentary Petrology”. W. H. Freedman and Company. San Francisco. U.S.A.. 564 p. BOUSQUIE, P., (1979): "Texture et porosité des roches calcaires". These doct. Univ. Pierre et Marie Curie et E.N.S.M.P Paris, $191 \mathrm{p}$

ESBERT, R. M.; MARCOS, R. M. (1983): "Las piedras de la Catedral de Oviedo y su deterioracion". Publ. Colegio Oficial de Aparejadores y Arquitectos Técnicos de Asturias. Gráficas Summa. Oviedo. $147 \mathrm{p}$.

ESBERT, R. M.; ORDAZ, J.; ALONSO, F. J.; VALDEON, L. (1984): “Influencia de las caracteristicas petrográficas en la durabilidad de areniscas utilizadas en monumentos”. I Congreso Español de Geologia. Tomo II, pp. 921-931.

FARMER, I. W., (1968): “Engineering properties of rocks”. Spon. Ltd. London. 180 p.

GOÑI, J. M.; GUILlEMIN, C.; RAGOT, J. P. y SIMA, A. (1970): "Méthodes d'étude de champ microfissural des minéraux et des roches au cours de leur alteration”. Revue de L'Industrie Minerále, Num. especial, pp. 40-51.

INTERNATIONAL SOCIETY FOR ROCK MECHANICKS (1979): “Comission on Standardization of Laboratory and Field Test”, Int. J. Rock Mech. Min. Sci. \& Geomech. Abstr.. Vol. 16. no 2. April. Pergamon Press. pp. 143-156.

MAMILLAN. M., (1981): "Connaissances actuelles des problemes de remontées d'eau par capillarité dans les murs". The Conservation of Stone. II. Proc. of the Int. Symp., Bologna. October. 1981. R. Rossi - Manaresi (Ed.). pp. 59-77.

MONTOTO. M.. (1983): "Petrophysics: The petrographic interpretation of the physical properties of rocks". Proc. 5th Int. Cong., Int. Soc. Rock Mechanics, Melbourne (Australia). April, Vol. B. pp. 93-98.

SUAREZ-VEGA, L. C. (1974): “Estratigrafia del Jurásico en Asturias” (1 y 2). Gráficas Summa. Oviedo, 369 p.

WALKeR, R. D.; PENCE, H. J.; HAZLETT, W. H. y ONG, W. J. (1969): “One cycle slow-freeze test for evaluation aggregate performance in frozen concrete". National Cooperative Highy Research Program Report, 65. 21 p. 